\title{
Far UV spectroscopy of the circumstellar environment of the Herbig Be stars HD 259431 and HD 250550
}

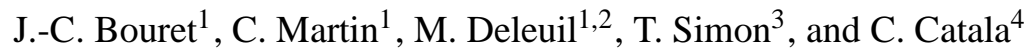 \\ ${ }^{1}$ Laboratoire d'Astrophysique de Marseille, Traverse du Siphon, BP 8, 13376 Marseille Cedex 12, France \\ e-mail: Claire.Martin@oamp.fr \\ ${ }^{2}$ Université de Provence, Centre de Mathématiques et d'Informatique, 39 rue J. Curie, 13453 Marseille Cedex 13, France \\ e-mail: Magali.Deleuil@oamp. fr \\ 3 Institute for Astronomy, University of Hawaii, 2680 Woodlawn Drive, Honolulu, HI 96822, USA \\ e-mail: simon@ifa.hawaii.edu \\ ${ }^{4}$ Observatoire de Paris-Meudon, CNRS, Paris, France \\ e-mail: Claude.Catala@obspm.fr
}

Received 9 May 2003 / Accepted 18 July 2003

\begin{abstract}
We present an analysis of FUSE spectra of HD 259431 and HD 250550, two young Herbig Be stars. Numerous absorption lines of $\mathrm{H}_{2}$ are seen in the spectrum of each star, revealing large amounts of this gas on the lines of sight. In addition, absorption lines from atomic species in different ionization and excitation states are also identified. We demonstrate that all these species probe the close circumstellar environment around the stars, with two or even three temperature components in HD 259431, as revealed by molecular gas analysis. Although favouring the flared disk scenario, our results do not rule out the possibility that we actually detect CS envelopes/halos around these stars.
\end{abstract}

Key words. stars: circumstellar matter - stars: pre-main sequence - stars: emission-lines, Be stars: individual: HD 259431, HD 250550

\section{Introduction}

Repeated observations have shown that the pre-main sequence (PMS) Herbig Ae/Be stars (HAeBes) share many properties in common with the classical T Tauri stars (CTTS), of which they are often considered to be the higher mass counterparts.

However, while the characteristics of the CTTS are generally thought to be linked to the presence of massive accretion disks (UV and IR excess emission, optical jets, asymmetric forbidden lines, ...), the nature of the close environment of the HAeBes remains a matter of considerable speculation.

Evidence is accumulating that a significant fraction of HAeBes present us with signatures of massive circumstellar (CS) disks. Millimeter-wave interferometric observations have revealed elongated structures around several HAeBes (Mannings \& Sargent 1997, 2000), while coronagraphic imaging in the near-IR, optical and UV domains has been performed successfully in a few cases (Pantin et al. 2000; Augereau et al. 2001; Grady et al. 2001). In this context, HAeBes have gained a lot of interest as the possible precursors of $\beta$-Pictoris and

Send offprint requests to: J.-C. Bouret, e-mail: Jean-Claude.Bouret@oamp.fr
Vega-type stars, whose CS debris disks are believed to host planetary bodies. This raises the possibility that the environment around the HAeBes represents an early phase of planet formation.

On the other hand, high spatial resolution mid-infrared observations have shown that the emission observed in HAeBes is generally not confined to optically thick disks but rather comes from more complex environments (Polomski et al. 2002). Besides, near IR speckle interferometry has demonstrated that halos on an arcsec scale occur much more commonly around HAeBes than around CTTS (Leinert et al. 2001); this latter result is fully compatible with the faster evolution of the more massive HAeBes. The same explanation may account for the fact that halos are much more common around B-type than A-type HAeBes. Similar findings were claimed by Natta et al. (2000) on the basis of mm interferometry.

The advent of far ultraviolet (FUV) observatories like FUSE now offers access to spectral lines that can directly and efficiently probe the circumstellar environment of HAeBes. This is especially true for lines of molecular hydrogen, which one would expect to be present if HAeBe stars have greatly extended CS disks and if the lines of sight to the stars pass through the disks. In that case, the Werner and Lyman electronic bands 
of $\mathrm{H}_{2}$ may be observable in absorption between $950 \AA$ and $1150 \AA$, projected against the UV continua of the stars. The detection of $\mathrm{H}_{2}$ absorption lines provides the most direct and most definitive measure of the gaseous content in the CS environment of HAeBes.

In addition, the FUSE domain contains several lines of highly ionized species like C III and O VI, which have been observed in the Herbig Ae stars AB Aur (Roberge et al. 2001) as well as HD 100546 and HD 163296 (Deleuil, private communication). These spectral lines directly probe regions of high temperatures above the stellar photosphere; the properties of these hot regions resemble those of the extended chromospheres modelled for a few HAeBes by Bouret et al. (1997) and Bouret \& Catala (1998) on the basis of optical and UV (IUE and HST/GHRS) data.

We present here FUSE spectra of HD 259431 and HD 250550, which were obtained for a program aimed at studying the occurence of hot and cold regions around HAeBes. In Sect. 2, we first present the observational material and the data analysis method we have used. Section 3 deals with the results we have obtained from our stellar modelling and a comparison with the known stellar properties. Section 4 is devoted to the analysis of the gaseous media on the line of sight to each star, whose results are discussed in Sect. 5. An extensive discussion will be given in Sect. 6 .

\section{Observations and data analysis}

HD 259431 and HD 250550 were selected for this analysis according to three major criteria: (i) a significant visual extinction $A_{\mathrm{v}}$, (ii) a high value of $V \sin i$ and (iii) a spectral type earlier than $\mathrm{A} 0$.

The first criterion indicates that the star is surrounded by a significant amount of material. This is a mandatory condition in order to search for the presence of $\mathrm{H}_{2}$ absorption lines between 950 and $1150 \AA$, which are expected if these stars are surrounded by massive circumstellar disks or envelopes.

The second criterion ensures that if such CS disks are present, the line of sight is likely to intercept the disk, since a high value of $V \sin i$ is indicative of a large inclination angle of the star's rotation axis.

Finally, the last criterion guarantees that the FUV continua of our target stars will be bright enough to serve as background illumination for the $\mathrm{H}_{2}$ spectral lines.

The two stars were observed with FUSE on October 1819, 2001, and October 15, 2001, respectively. The data were obtained with the $30^{\prime \prime} \times 30^{\prime \prime}$ LWRS aperture, covering the wavelength range 905-1187 $\AA$ at a spectral resolution of $R \approx$ 15000 . The spectra were reduced and calibrated using version 2.2.2 of CalFUSE, the FUSE pipeline processing software. The total exposure time was $16.5 \mathrm{ks}$ for HD 259431 and $9 \mathrm{ks}$ for HD 250550, split into thirteen and three subexposures, respectively.

For HD 259431, data for LiF2A segment do not reproduce well from one subexposure to the next, presumably because of a drift of the star over the course of the observation. On the other hand, the flux level among the subexposures is more consistent in the LiF1B channel but the latter is affected by the so-called worm artifact, which produces a broad depression in the spectra and modifies the actual flux distribution longward of $1150 \AA$. To overcome this problem, we used the LiF1B data below $1150 \AA$ only, while above that wavelength we used LiF2A segment scaled to the flux level in IUE spectra of HD 259431 (large aperture SWP data) where the two spectral energy distributions overlap (over $30 \AA$ approximately).

For HD 250550, the LiF2A and LiF1B segments have roughly the same flux level, although the LiF1B data are also clearly affected by the worm artifact. Hence, the LiF1B spectrum was used only to confirm the presence of the lines identified in the LiF2A channel, not for the line profile analysis.

The co-added spectra of HD 259431 were rebinned by 5 pixels, in order to increase the signal-to-noise ratio without degrading the resolution. The resulting signal-to-noise ratio for the spectrum of HD 259431 is $S / N \sim 33$ per spectral resolution element at $1150 \AA$, with detectable stellar flux down to $955 \AA$. Similarly, the co-added spectra of HD 250550 were rebinned by 10 , which yielded a signal-to-noise ratio of $S / N \sim 16$ per resolution element at $1150 \AA$; the stellar continuum is well detected down to wavelengths shortward of $1000 \AA$.

In order to ensure as accurate a wavelength calibration as possible, the individual subexposures in each FUSE channel were aligned using a linear cross-correlation procedure prior to co-addition. We used several photospheric lines as predicted by models with appropriate parameters for each star (see details in Sect. 3) to set the absolute wavelength scales of the spectra in the stars' reference frames. We corrected for offsets of $-1 \mathrm{~km} \mathrm{~s}^{-1}$ for HD 259431 and $-10 \mathrm{~km} \mathrm{~s}^{-1}$ for HD 250550, respectively; the resulting uncertainty in the wavelength calibration is $1 \mathrm{~km} \mathrm{~s}^{-1}$ for HD 259431 and $5 \mathrm{~km} \mathrm{~s}^{-1}$ for HD 250550.

The FUSE spectra of HD 259431 and HD 250550 exhibit many narrow absorption lines, which correspond to electronic transitions from the ground level or excited fine structure levels of various atomic species in different ionization states. Much broader lines corresponding to electronic transitions of molecular hydrogen are observed in both spectra.

So as to better assess the nature of these lines and to help disentangle purely stellar (i.e. photospheric) features from circumstellar and/or interstellar (IS) ones, we undertook a detailed modelling of the photospheres of both stars, with state-ofthe-art photospheric models (see Sect. 3.1). This photospheric modelling was also used to properly estimate the stellar continuum level, necessary in particular for the subsequent analysis of the CS/IS spectral lines.

\section{Stellar properties}

Stellar radial velocities in the heliocentric rest frame were measured by Finkenzeller \& Jankovics (1984): $V_{\mathrm{rad}}=+43 \mathrm{~km} \mathrm{~s}^{-1}$ and $V_{\text {rad }}=+31 \mathrm{~km} \mathrm{~s}^{-1}$ for HD 259431 and HD 250550, respectively. The same authors also gave measurements of the radial velocities of the molecular clouds associated to each star: $V_{\mathrm{MC}}=+19.9 \mathrm{~km} \mathrm{~s}^{-1}$ for HD 259431 while $V_{\mathrm{MC}}=+16.2 \mathrm{~km} \mathrm{~s}^{-1}$ for HD 250550.

Projected rotation velocities of these stars were measured by Böhm \& Catala (1995): $V$ sin $i=90 \pm 8 \mathrm{~km} \mathrm{~s}^{-1}$ and 
$V \sin i=110 \pm 9 \mathrm{~km} \mathrm{~s}^{-1}$ for HD 259431 and HD 250550, respectively. These values agree within the error bars with those of Finkenzeller (1985).

On the other hand, various estimates of the fundamental parameters of HD 259431 and HD 250550 have been proposed over the past 20 years. Spectral types and $T_{\text {eff }}$ as different as B5 V, $15300 \mathrm{~K}$ (Garrison 1978), or B1 V, $25700 \mathrm{~K}$ (van den Ancker et al. 1998), have been quoted for HD 259431. As a consequence, estimates of the visual extinction towards this star range widely from $A_{\mathrm{v}}=0.83$ (Valenti et al. 2003) to $A_{\mathrm{v}}=1.61$ (van den Ancker et al. 1998). HD 250550 has been claimed to be either B9 V, $12300 \mathrm{~K}$ (Garrison 1978), or B4-5 V, $15800 \mathrm{~K}$ (van den Ancker et al. 1998). Here again, the estimates of $A_{\mathrm{v}}$ range from 0.5 (Hillenbrand et al. 1992) to 0.74 (Valenti et al. 2003).

These significant discrepancies have motivated a thorough modelling of the stars' photospheres. This modelling enabled us to derive H I column densities on the lines of sight by estimating the stellar contribution to the observed Ly $\alpha$ line profile. This is an important step for our analysis of the CS/IS gas component seen in our FUSE spectra. To our knowledge, atomic hydrogen column densities are not available in the literature for either star.

To help in this task, we extracted spectra from the IUE archives as well as from the spectral atlas of PMS stars compiled by Valenti et al. (2000, 2003), which includes co-added low-resolution spectra for both the short-wavelength range

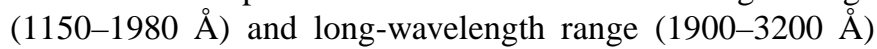
of IUE. Processing and analysis procedures for each range are described in Valenti et al. (2000) and Valenti et al. (2003) respectively.

Concerning these IUE data, we note that the highresolution spectra from the archives clearly show that the spectrum of HD 250550 is much richer in CS absorption features than that of HD 259431. It is also worth mentioning that HD 259431 shows a shallower $2200 \AA$ absorption feature. Sitko (1981) interpreted this as a clue that graphite grains larger than $0.02 \mu$ are present around this star, since smaller graphite dust particles, in large enough amount, would produce a deep and sharp absorption trough around $2200 \AA$.

\subsection{Determination of photospheric parameters}

Our targets are classified as B-type stars and their effective temperatures are most likely high enough for NLTE processes to become important in their photospheres. Accurate determination of the stars fundamental parameters, as well as photospheric line profiles fitting, therefore require modelling with codes that explicitly allow for departures from LTE in the atmospheres. Since modelling with such codes is a highly time-consuming process, the following strategy was adopted to speed up this step.

Photospheric models were initially extracted from the Kurucz grid of LTE models (Kurucz 1994). The sub-grid thus obtained samples the parameters space $11000 \mathrm{~K} \leq T_{\text {eff }} \leq$ $20000 \mathrm{~K}$ with $500 \mathrm{~K}$ steps and $4.0 \leq \log g \leq 4.75$ with 0.25 dex steps. Synthetic spectra were calculated in NLTE using the SYNSPEC package of Hubeny \& Lanz (1995). This code allows for an approximate NLTE treatment of lines with LTE photospheric models (see SYNSPEC's User's Guide at http://tlusty.gsfc.nasa.gov/ for details). The result of this first stage is to determine the LTE model giving the best fit to the observed spectra.

In a second step, NLTE photospheric models were constructed with the code TLUSTY (Hubeny \& Lanz 1995), using parameters from the LTE step as a starting point. This helped to greatly reduce the parameter space we needed to explore with NLTE models.

The main assumptions of TLUSTY are a plane-parallel geometry, hydrostatic equilibrium, and radiative equilibrium. Departures from LTE are explicitly allowed for a large set of chemical species and arbitrarily complex model atoms. Line opacity is treated in detail using an Opacity Sampling technique. For this work we used close to 20000 frequency points over the whole spectrum, thereby ensuring a spectral resolution that is slightly higher than the observed data. Our blanketed, NLTE model atmospheres include about 530 individual explicit atomic levels from 21 ions (H I, He I, C I-III, N I-III, O I-III, Mg I-II, Al I-II, Si I-III, Fe I-III). We computed models with a solar composition. All model atmospheres assume a helium abundance, $Y=\mathrm{He} / \mathrm{H}=0.1$ by number, and a microturbulent velocity, $\xi_{\mathrm{t}}=2 \mathrm{~km} \mathrm{~s}^{-1}$.

TLUSTY solves for the full photospheric structure, providing the temperature and density stratification and the NLTE populations. A detailed synthetic spectrum is then calculated with SYNSPEC, varying if necessary the abundance of individual species (e.g., the N/C abundance ratio), or the microturbulent velocity. However, we have always imposed that the photospheric turbulent velocity remains smaller than the photospheric speed of sound (typically, $v_{\text {sound }} \leq 12 \mathrm{~km} \mathrm{~s}^{-1}$ ) for temperatures around $15000 \mathrm{~K}$.

The fundamental parameters of our program stars were determined considering the dereddened FUSE and IUE spectral energy distributions and line ratios between successive ionization states of various chemical species. Note that FUV spectra are insensitive to $\log g$. Therefore, there is a degeneracy in the determination of $\log g$ from our FUV modelling, which we reduced by using stellar masses and radii obtained by other means (see below).

To compare synthetic spectra with FUSE and IUE data, we chose to normalize the synthetic spectra at $3000 \AA$ rather than in the $V$ band. This reduces the effects of errors in the optical photometry, and helps better determine the stars' extinction laws in the UV, as shown by Boggs \& Böhm-Vitense (1989) and Thé et al. (1996). We refer the reader to those papers for more details about the method and its results.

The observed spectra were dereddened using the Cardelli, Clayton \& Mathis (Cardelli et al. 1989) extinction law and adopting $R_{\mathrm{V}}=A_{\mathrm{v}} / E(B-V)=3.1$ in a first step. As noted earlier, very different values for the visual extinction have been published in the past. Most of them are based on photometric measurements in the visible and IR (e.g., Hamann \& Persson 1992; Hillenbrand et al. 1992; van den Ancker et al. 1998), and therefore their relevance for the UV and FUV extinction law may be questionable. On the other hand, 
Valenti et al. (2003) published $A_{\mathrm{v}}$ values obtained by comparing IUE spectra of HD 250550 and HD 259431 with those of stars of similar spectral types and very low extinction. We adopted these $A_{\mathrm{v}}$ values for our work but also allowed for an anomalous extinction law in the FUV, in other words, we let $E(B-V)$ and $R_{\mathrm{v}}$ vary until a good match was obtained between the theoretical and observed spectra. It should be noted here that the adopted value for $E(B-V)$ has no effect on the analysis of the star's environment that we perform using the numerous absorption lines (atomic and molecular) in the FUSE spectra (Sect. 4). We checked that carefully by comparing column densities measured on spectra corrected and not corrected for reddening, respectively.

Once $T_{\text {eff }}$ and $A_{\mathrm{v}}$ are determined from the steps outlined above (error bars on $T_{\text {eff }}$ are determined from a $\chi^{2}$ procedure from the fits), $\log g$ is obtained by two independant methods. First, synthetic spectra with values of $T_{\text {eff }}$ and $A_{\mathrm{v}}$ as determined above and with various values of $\log g$ are compared with the observed spectra, until a best fit is achieved. Second, stellar luminosities and radii are calculated from $T_{\text {eff }}$ and $A_{\mathrm{v}}$ using HIPPARCOS parallaxes (van den Ancker et al. 1998; Bertout et al. 1999) and bolometric corrections from Flower (1996). Stellar masses and ages are then evaluated from the evolutionary tracks of Palla \& Stahler (1993), which were kindly provided to us by Dr. L. Testi. The values of $\log g$ determined from stellar masses and radii are in remarkable agreement with those determined spectroscopically.

A spectral type for each star is determined from its effective temperature, by using the Schmidt-Kaler (1982) calibration. The error bars on $T_{\text {eff }}$ are, in all cases, well within the dispersion around the mean effective temperature ascribed to a given spectral type by Schmidt-Kaler (1982). We are therefore entirely confident that the spectral types we propose are indeed appropriate for the stars' effective temperatures. The remaining uncertainty lies in the Schmidt-Kaler (1982) calibration, but we have confirmed that stellar parameters from other sources, e.g., those listed in Bessell et al. (1998) still yield the same spectral types.

The stellar parameters obtained from this analysis are listed in Table 1.

\subsection{HD 259431}

As shown in Fig. 1, the dereddened spectral energy distribution in the FUSE wavelength range is well reproduced by a TLUSTY model with values of $T_{\text {eff }}$ and $\log g$ as determined in the previous section once convolved with a rotational velocity of $V \sin i=95 \pm 10 \mathrm{~km} \mathrm{~s}^{-1}$. Note that the detection of the stellar continuum down to $\sim 955 \AA$ is indeed expected for a star with $T_{\text {eff }}=15900 \pm 200 \mathrm{~K}$. We also checked that the same model yields a very good fit to the $I U E$ data we obtained from Valenti et al. (2003) and that the hydrogen and helium line profiles predicted by the best fit model are fully consistent with observed profiles in the visible range (Finkenzeller \& Jankovics 1984). Therefore, we argue that the stellar parameters provided by the best fit model for HD 259431 (Fig. 1) are the most realistic and accurate ones to-date. Almost all the photospheric features visible in the FUSE spectrum are reproduced by the model, clearly highlighting the CS and/or IS components. Note, for instance, the prominent $\mathrm{H}_{2}$ bands in the spectrum, superimposed on the photospheric flux. This demonstrates the power of using a realistic photospheric modelling to estimate the proper level of the stellar continuum.

The higher value of $R_{\mathrm{v}}$ (3.4) we obtain compared to that of Valenti et al. (2003), for a very similar $A_{\mathrm{v}}=0.88$, clearly indicates that the extinction law departs from the standard interstellar law. This is not surprising for a young Herbig Be star like HD 259431, where CS dust grains with a size distribution differing from that of the average IS medium strongly contribute to scatter and screen the light from the star.

As already pointed out by Grady et al. (1988), IUE spectra of HD 259431 show P Cygni profiles in the Mg II h \& $\mathrm{k}$ lines, which is a sign of a significant stellar wind. At shorter wavelengths, IUE spectra also show strong absorption lines of C IV $\lambda \lambda 1548-1550$ and Si IV $\lambda \lambda 1394-1402 \AA$, blueshifted by up to $-400 \mathrm{~km} \mathrm{~s}^{-1}$. This demonstrates the existence of regions heated to temperatures much higher than $T_{\text {eff }}$ in the wind of HD 259431. This is similar to what is known of several other Herbig Ae/Be stars (e.g., HD 250550 and the prototypical Herbig Ae star AB Aur); the usual interpretation involves an extended chromosphere embedded in a stellar wind, which is expected to produce highly ionized species.

A direct comparison of our model with the FUSE spectrum makes clear that the observed line of the C III multiplet UV 4 at $1175 \AA$ is slightly broader and stronger than the pure photospheric contribution. Given the $V \sin i$ of the star, the six individual components of the multiplet (at $\lambda 1174.93$, $\lambda 1175.26, \lambda 1175.59, \lambda 1175.71, \lambda 1175.98$, and $\lambda 1176.37)$ are blended together. Measured with respect to the rest wavelength of the bluest component, the extra absorption appears to be blueshifted by $-305 \mathrm{~km} \mathrm{~s}^{-1}$, which suggests that we are detecting a chromosphere/wind contribution to the line.

A weak emission line from C III $977 \AA$ may also be present, superimposed on the stellar (photospheric) absorption line (see Fig. 1). However, a small change of -0.02 mag in the $E(B-V)$ value we have used would shift the photospheric model above this weak feature, thus invalidating this detection. Since our determination of $E(B-V)$ is $0.26_{-0.02}^{+0.01}$, we cannot go into much details about this line and simply state that if real, the line shape is consistent with a line formation occuring in an extended, hot, rotating envelope.

Since IUE spectra clearly show strong C IV resonance lines in absorption (Grady et al. 1988), a conspicuous emission from the C III $\lambda 977$ resonance line would be expected in the FUV spectrum of HD 259431. Using a qualitative approach of the line formation, we searched for the physical conditions required to get weak (if any) C III $\lambda 977$ emission and strong C IV $\lambda \lambda 1548-1550$ lines in absorption. We found that the C IV resonance lines form in emission at temperatures higher than $90000 \mathrm{~K}$ for electron densities typical of Herbig Be stars winds $\left(n_{\mathrm{e}} \approx 1 \times 10^{12}\right)$. On the other hand, at slightly lower temperatures, these lines still form in absorption while C III is mostly depleted, and only a very weak $\lambda 977$ line can be produced, as observed. 
Table 1. Astrophysical parameters of the stars, as deduced from the modelling of their FUSE spectra. $V_{\text {rad }}$ are measured in the heliocentric rest frame (Finkenzeller \& Jankovics 1984).
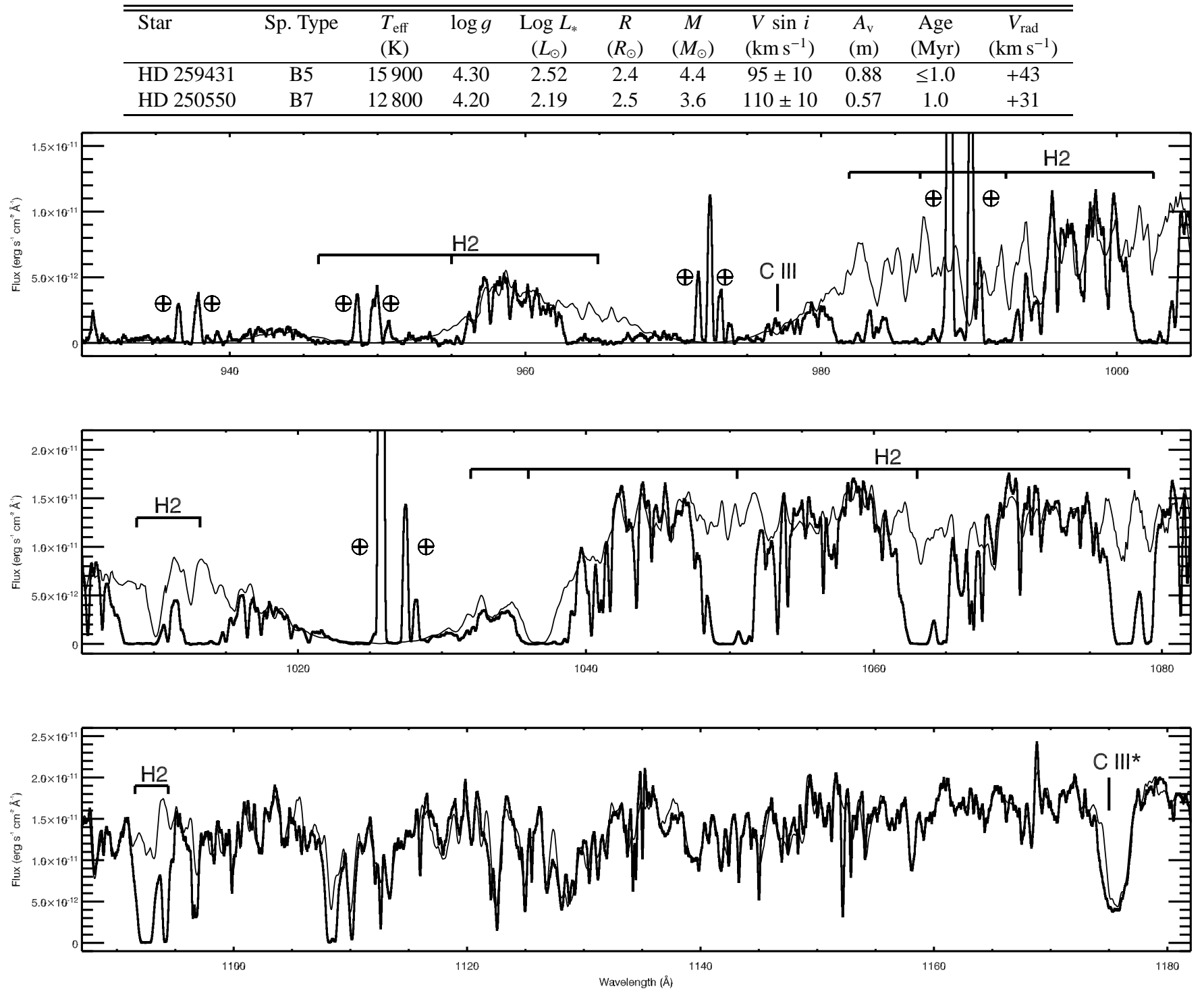

Fig. 1. Comparison between the dereddened HD 259431 FUSE spectrum (thick line) and a TLUSTY model with $T_{\text {eff }}=15900 \mathrm{~K}, \log g=4.3$ (thin line). The model is convolved with $V \sin i=95 \mathrm{~km} \mathrm{~s}^{-1}$. Airglow lines are indicated with $\oplus$ symbols. The principal prominent absorption troughs caused by molecular hydrogen are indicated throughout the spectrum.

Going to higher ionization potentials, no emission lines from the O VI $\lambda \lambda 1032-1038$ resonance doublet are apparent. With the upper error bar on $E(B-V)$, we are still confident that no O VI lines are detected in HD 259431, as the corrected flux is still below the model's flux in the doublet spectral range. The absence of high temperature emission may be due to overlying absorption from molecular hydrogen; the amount of absorption we find in Sect 4.2.1 likely is large enough to suppress emission components in both lines, $\lambda 1032$ and $\lambda 1038$ (see e.g. Roberge et al. 2001 in the case of AB Aur for a lower molecular column density). On the other hand, since temperatures lower than $90000 \mathrm{~K}$ are favoured by the $\mathrm{C}$ IV resonance lines being in absorption, it is unlikely that $\mathrm{O}$ VI is actually present in the star's envelope.

\section{3. $H D 250550$}

As shown in Fig. 2, the observed dereddened spectral energy distribution in the FUSE wavelength range is well reproduced by a TLUSTY model with values of $T_{\text {eff }}$ and $\log g$ as determined in Sect. 3.1, once convolved with a rotational velocity of $V \sin i=110 \pm 10 \mathrm{~km} \mathrm{~s}^{-1}$. Note the detection of the stellar continuum down to $\sim 1000 \AA$, as expected for a star with $T_{\text {eff }}=12800 \pm 200 \mathrm{~K}$, and the strong absorption features, indicated with vertical arrows, which are much stronger than predicted by the photospheric model. The origin and nature of these prominent features are discussed below. We also checked that the same model yields a very good fit to the $I U E$ data we obtained from Valenti et al. (2003). 

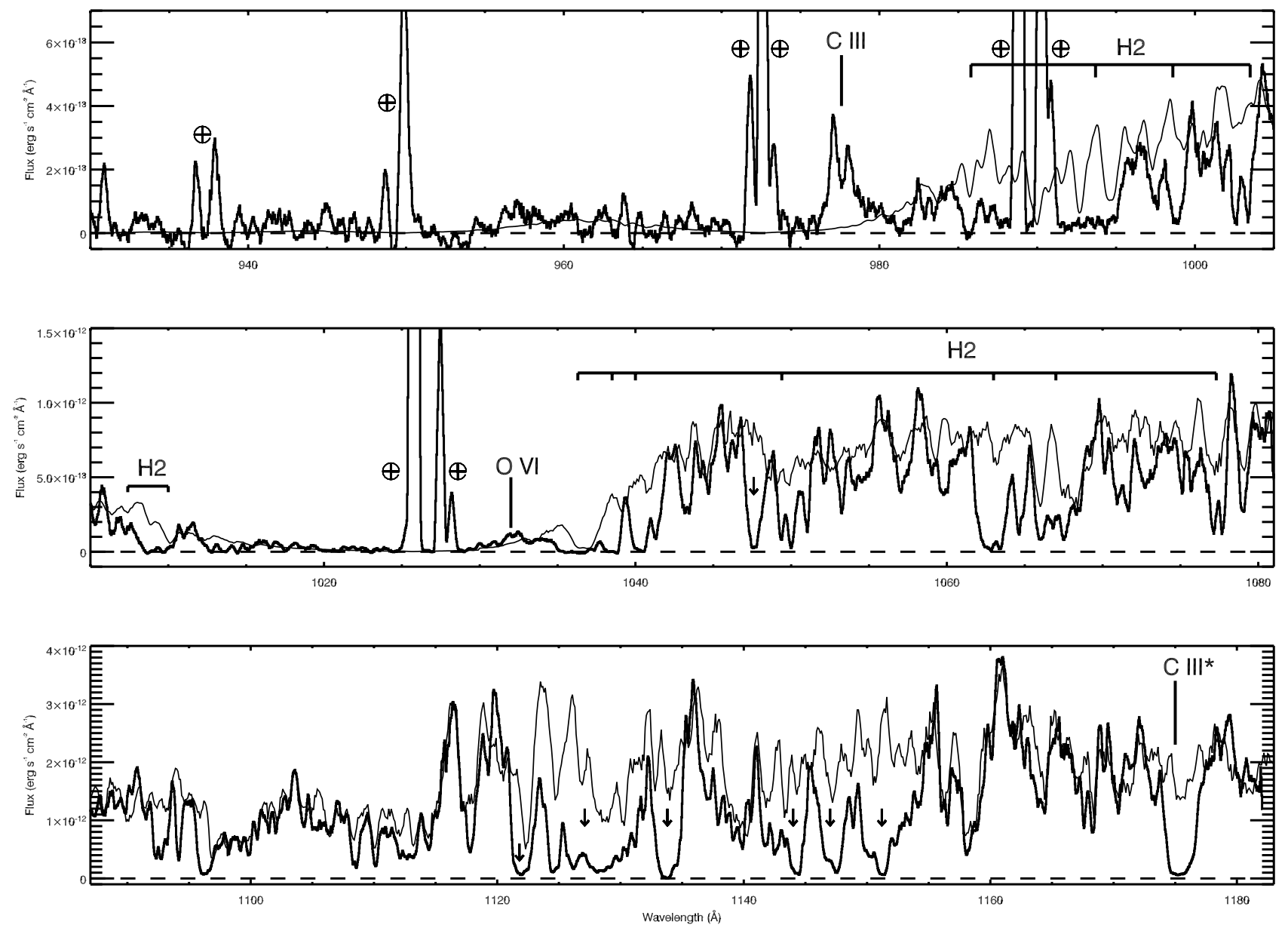

Fig. 2. Comparison between the dereddened HD 250550 FUSE spectrum (thick line) and a TLUSTY model with $T_{\text {eff }}=12800 \mathrm{~K}, \log g=4.2$ (thin line). The model is convolved with $V \sin i=110 \mathrm{~km} \mathrm{~s}^{-1}$. Airglow lines are indicated with $\oplus$ symbols. The principal prominent absorption troughs due to $\mathrm{H}_{2}$ are indicated throughout the spectrum. The presence of wind features due to Fe II and/or N I is evidenced by vertical arrows in the LiF2A channel (between 1090 and $1180 \AA$ A).

From the model, we derived an extinction law with $E(B-$ $V)=0.22 \pm 0.02$ and $R_{\mathrm{v}}=2.6$, and thus $A_{\mathrm{v}}=0.57$. The extinction towards HD 250550 is therefore very different from interstellar extinction. Our value for $A_{\mathrm{v}}$ is in good agreement with the value determined by Hillenbrand et al. (1992) from IR and optical photometry, while our $E(B-V)$ is similar to the value implied by the $A_{\mathrm{v}}$ of Valenti et al. (2003), assuming $R_{\mathrm{v}}=3.1$.

In the FUSE spectrum, a clear emission line due to C III $977 \AA$ is present in the SiC2A channel (see Fig. 2). The line is double-peaked, having a well-marked central depression that is redshifted by $+300 \mathrm{~km} \mathrm{~s}^{-1}$ with respect to the rest wavelength in the star's rest frame. The blue peak is redshifted by $+35 \mathrm{~km} \mathrm{~s}^{-1}$, while the red one is at $+375 \mathrm{~km} \mathrm{~s}^{-1}$. A broad emission wing extends farther to the red, up to $\approx 980 \AA$ (i.e. $+1000 \mathrm{~km} \mathrm{~s}^{-1}$ ), making the total width of the line $\Delta V \approx$ $1350 \mathrm{~km} \mathrm{~s}^{-1}$. While a detailed modelling of the processes leading to the formation of emission in C III is beyond the scope of the present paper, the observed behavior can be interpreted in the context of the chromospheric scenario proposed by Bouret $\&$ Catala (1998) to account for the formation of P Cygni profiles at $\mathrm{H} \alpha$ and $\mathrm{Mg}$ II $\mathrm{h} \& \mathrm{k}$, and $\mathrm{C}$ IV $\lambda \lambda 1548-1550$ lines in absorption. The model assumes that an extended chromosphere of moderate temperature $\left(T_{\max }=20000 \mathrm{~K}\right)$ is embedded in a strong stellar wind $\left(\dot{M}=3 \times 10^{-8} M_{\odot} \mathrm{yr}^{-1}, V_{\infty}=450 \mathrm{~km} \mathrm{~s}^{-1}\right)$. In the model, the temperature drops down very steeply after the chromospheric region, and the outermost regions of the wind are therefore isothermal $\left(T_{\text {out }}=3000 \mathrm{~K}\right)$. In this framework, the blue peak $\left(V=+35 \mathrm{~km} \mathrm{~s}^{-1}\right)$ might correspond to a wind emission while the redmost peak $\left(V=+375 \mathrm{~km} \mathrm{~s}^{-1}\right)$ would be produced by accretion onto the star. Indeed, we have checked that, in the chromosphere + wind model by Bouret \& Catala (1998), the line forms in emission and reproduces well the observed blue peak of the line. As a matter of fact, this blue peak corresponds to the emission component of a $\mathrm{P}$ Cygni profile whose blueshifted absorption component is not seen because of the very low level of the continuum below $977 \AA$. The red peak and its broad wing, on the other hand, suggest that an active phase of (magnetospheric) accretion (e.g., Muzerolle et al. 2001) was underway at the time of our FUSE observation.

A comparison of the TLUSTY model and the LiF2A spectrum near $1175 \AA$ shows clearly that the observed C III (multiplet 4) line is mostly non-photospheric. The line is very broad, with $\Delta V \approx 1000 \mathrm{~km} \mathrm{~s}^{-1}$. Given the high $V \sin i$ of the star, the six individual components of the multiplet are blended together. Measured with respect to the rest wavelength of the bluemost and redmost component, the blue and red side of 
the observed profile are located at $\Delta V_{\text {blue }} \approx-360 \mathrm{~km} \mathrm{~s}^{-1}$ and $\Delta V_{\text {red }} \approx+270 \mathrm{~km} \mathrm{~s}^{-1}$, respectively. The chromosphere + wind model, established through the modelling of CIV $\lambda \lambda 1548$ 1550, $\mathrm{H} \alpha, \mathrm{Mg}$ II $\lambda \lambda 2795-2802$, predicts that the line should form as a blueshifted absorption line. The blue edge of this profile, which indicates the wind's maximal velocity in the line formation region, is at $-320 \mathrm{~km} \mathrm{~s}^{-1}$. This is in fairly good agreement with the velocity measured for $C$ III $\lambda 1175$, given that no adjustments were made to the model, for the specific case of these new data. On the other hand, the theoretical line is not as strong as the observed one, which suggests either that C III in excited states is not predicted correctly by the chromospheric model or that significant amounts of this ion are located on the line of sight and are produced by different mechanisms.

The $\lambda 1032$ component of the O VI resonance doublet is clearly present in the LiF1A spectrum of HD 250550, while the $\lambda 1038$ component is completely suppressed by broad $\mathrm{H}_{2}$ absorption lines near $1037 \AA$ and $1039 \AA$. The complex shape of the $\lambda 1032 \mathrm{O}$ VI line cannot result from the superposition of $\mathrm{H}_{2}$ lines on a more regular, but clearly non-Gaussian emission line profile, as these $\mathrm{H}_{2}$ lines correspond to high $J$-levels $(J>4)$ that are not seen in other parts of the spectrum. The velocity shifts of the two peaks of the profile and the central trough, $\Delta V_{\text {blue }} \approx+90 \mathrm{~km} \mathrm{~s}^{-1}, \Delta V_{\text {trough }} \approx+400 \mathrm{~km} \mathrm{~s}^{-1}$ and $\Delta V_{\text {red }} \approx+630 \mathrm{~km} \mathrm{~s}^{-1}$, respectively, make the observed line profile reminiscent of the C III $\lambda 977$ profile. On the other hand, it is rather unlikely that the O VI line is optically thick, as it should be if it was indeed formed in the massive wind of HD 250550. The similarity with the profile of C III $\lambda 977$ is likely fortuitous and the line may rather form in magnetically channelled accretion onto the stellar surface. In this respect, it is worth noting that our analysis of the absorption lines of Fe II and Fe III present in the HD 250550 FUSE spectrum (see Sect. 4.1.2) shows that the ionization ratio between these two ions is such that temperatures higher than $20000 \mathrm{~K}$ are ruled out for the wind; this is an independant confirmation that the chromospheric model of Bouret \& Catala (1998) is an adequate description of HD 250550. Therefore, a wind origin for the $\mathrm{O}$ VI line is unlikely since temperatures of a few $10^{5} \mathrm{~K}$ are required to ionize $\mathrm{O} \mathrm{V}$.

The presence of a strong stellar wind is also further strengthened by the strong absorption features in LiF2A segment. These features most likely correspond to fine structure transitions of Fe II formed in the wind of HD 250550. In IUE data, the Fe II lines around $2600 \AA$ show the same structure (see Fig. 3), namely a broad, blueshifted, absorption component. An additional narrow component is observed at the star's velocity and likely corresponds to CS gas. The IUE lines are easier to interpret as they are less severely blended with each other than those in the FUSE range. The maximum blueshift measured on the Fe II lines, both in FUSE and IUE spectra, is $\Delta V \approx-350 \mathrm{~km} \mathrm{~s}^{-1}$, i.e., fully consistent with a line formation taking place in the wind. Note that one of these broad wind features corresponds to the N I triplet near $\lambda 1135$, the others to Fe II excited and fine structure lines. Because of the severe blends we have mentioned, the modelling of Fe II lines formation in stellar winds is a complex task, which we plan to undertake in a forthcoming paper. Finally, we checked carefully that

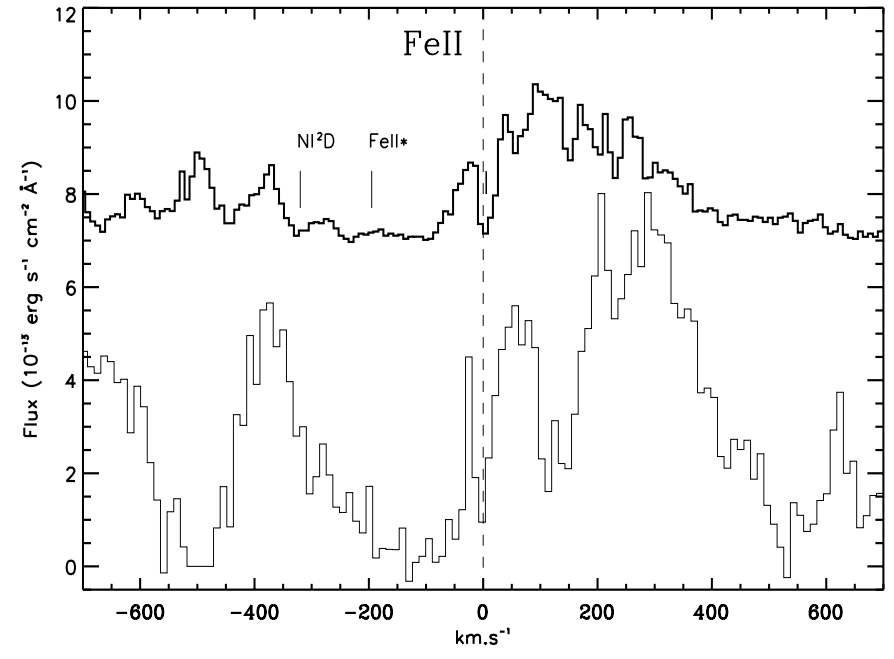

Fig. 3. Fe II lines observed by FUSE at $1145 \AA$ (top curve) and IUE at $2586 \AA$ (bottom curve). The $x$-axis shows velocities in the star's rest frame. The $y$-axis is in units of $10^{-13} \mathrm{ergs} \mathrm{cm}^{-2} \mathrm{~s}^{-1} \AA^{-1}$ (fluxes not corrected for reddening); the FUSE spectrum was shifted upward by +7 for the sake of clarity. The lines show the same overall structure, namely a blueshifted, broad, component and a narrow component observed at the star's velocity (dashed line). Fe II* and $\mathrm{N} \mathrm{I}^{2} \mathrm{D}$ lines are blended with the Fe II line in the FUSE data.

if some of the other atomic species detected in the spectrum (see Sect. 4.1.2) present such wind components in addition to the CS components at the star's velocity, we are unable to detect them as they all fall within those produced by Fe II. Therefore, we cannot rule out the possibility that these species also form partly in the wind of HD 250550.

\section{Analysis of the stellar environment}

Once the purely stellar analysis was completed, we undertook a thorough analysis of the narrow absorption lines present in the two spectra that are not accounted for by the photospheric models. These absorption lines correspond to electronic transitions from the ground level, as well as fine structure or excited levels. The spectra also show numerous lines from molecular hydrogen.

The analysis of the absorption lines in the observed spectra was performed using the OWENS package, written by Dr. M. Lemoine (see Lemoine et al. 2002). OWENS is a versatile profile fitting program, which treats all the lines of a given ion simultaneously, throughout all of the FUSE spectral channels, when necessary. Intrinsic line profiles for atomic/ionic species are modelled by Voigt profiles and are further convolved with a Gaussian to simulate the instrumental line spread function. The continuum is locally fitted with polynomials (of up to 14th degree, if necessary). We used FUV wavelengths and oscillator strengths tabulated by Morton (2000). Electronic transitions of $\mathrm{H}_{2}$ were modelled using data tabulated in Abgrall et al. (1993a,b), Abgrall et al. (2000) and Balakrishnan et al. (1999).

For each ion and/or molecule (CS or/and IS), $\chi^{2}$ fitting of unsaturated and/or damped lines provides estimates of the corresponding column densities. Radial velocities can be 
measured on unsaturated lines, while intrinsic line widths (denoted herein by $b$ ) are usually determined from saturated lines. Our estimates of these quantities are tabulated adopting conservative $2 \sigma$ error bars.

For each element and ionization stage, we have measured the column density in the different energy levels, on the basis of the profile fitting procedure described above. The procedure is illustrated in Figs. $4 \mathrm{a}$ and $4 \mathrm{~b}$ for the specific case of Fe II in HD 259431 and HD 250550, respectively.

Tables 2 and 3 summarize our results. Detailed comments on the individual species follow below.

\subsection{Atomic species}

\subsubsection{HD 259431}

- NI - No column density could be measured for NI. Although the full resonance triplet is seen, each one of the three components is saturated. No transitions from excited levels are seen; this could be a consequence of the higher $T_{\text {eff }}$ of HD 259431, which perhaps shifts the ionization balance towards N II. N II lines in the FUSE spectral range that might confirm this speculation coincide with strongly saturated photospheric lines.

We measured a radial velocity for the three saturated but still reasonably sharp lines of the resonance triplet; these lines are redshifted by about $15 \mathrm{~km} \mathrm{~s}^{-1}$ (see Table 4).

- O I - Resonance lines and fine structure lines from this ion fall in the FUSE bandpass. The flux level and signal-tonoise ratio of the spectrum in the LiF1A are high enough that we can detect absorption lines from the three lowest energy levels. We also detect the metastable $\lambda 1152.15$ line from the first excited level (at $15867.86 \mathrm{~cm}^{-1}$ ). All O I lines are saturated. Line fitting with one component yields damped lines and very high values for the column densities $\left(10^{2}\right.$ times higher than $\mathrm{H}_{2}$ column densities) which are incompatible with the general properties of CS or IS media. Our $\chi^{2}$ tests on the column densities favour two component models.

- P II - Two resonance lines and several lines from the first two fine structure levels of P II are observed. For the ground level and the fine structure level at $469.12 \mathrm{~cm}^{-1}$, the column densities were estimated from the unsaturated lines at $1142.9 \AA$ and $1159 \AA$. Saturated lines arising from the same levels were used to derive $b$ value. All of the lines from the first fine structure level (at $164.90 \mathrm{~cm}^{-1}$ ) are unsaturated.

- ArI - Two ArI resonance lines fall in the FUSE spectral range and are observed in HD 259431. Two components are required to fit the lines (same comments as for $\mathrm{OI}$ ), but both lines are saturated, preventing accurate measurements of the radial velocity and column density.

- Mn II - Three resonance lines of Mn II are observed within the LiF2A channel. These lines are weak and unsaturated. One of them, at $1164.208 \AA$, is characterized by a larger radial velocity shift than the other two. The same phenomenon is observed in the FUSE spectra of HD 250550 (see below) and HD 100546, another Herbig star (Deleuil, private communication). The discrepancy strongly suggests that the theoretical wavelength of this transition may be wrong.

- Fe II - Several absorption lines arising from ground and excited levels up to $977 \mathrm{~cm}^{-1}$ are observed (see Fig 4a), allowing a complete analysis of this ion in terms of column densities, radial velocity and $b$.

- Fe III - This ion is, roughly, as abundant as Fe II. The presence of such an ion in the CS environment of a B5 V star implies that a significant source of non-radiative heating exists in the vicinity of the star. This ion must be produced by collision processes involving relatively dense gas. Indeed, the photoionization threshold from Fe II to Fe III is high enough $(16.19 \mathrm{eV})$ that it requires photons shortward of $766 \AA$, where the stellar flux of a $15900 \mathrm{~K}$ star like HD 259431 is virtually zero.

\subsubsection{HD 250550}

- N I - The strongest line of the resonance triplet, $\lambda 1135$, is observed. The other two components are canceled by strong saturated lines from the stellar wind. Numerous lines arising from the first two excited levels are seen. Because the only resonance line available is saturated, the total column density of $\mathrm{N}$ I we derived from the excited levels must be considered as a lower limit.

- OI - None of the few OI resonance and fine structure lines within the FUSE wavelength range is observed in HD 250550. This is also true for the $\lambda 1152.15$ line from the first excited level, which lies at the bottom of a highly saturated wind line from Fe II (see below). The detection of [O I] $6300 \AA$ line by Böhm \& Catala (1994) suggests that this ion must nevertheless be present in the environment of HD 250550, but unfortunately we cannot claim a detection on the basis of our FUSE data.

- P II - Same comments as for HD 259431.

- $\mathrm{Cl}$ I and Cl II - In contrast to HD 259431, we detect fairly large amounts of $\mathrm{Cl}$ I and $\mathrm{Cl}$ II in the spectrum of HD 250550.

- ArI - The two ArI resonance lines in the FUSE domain are observed. One is saturated, the second is not. This is a favourable situation that allows us to accurately estimate the gas column density, the intrinsic line width and radial velocity from only two lines.

- Mn II - Three weak and unsaturated resonance lines of Mn II are observed. Same comments as for HD 259431.

- Fe II - Because of the presence of the strong stellar wind, as described in Sect. 3.3, we have not used the photospheric model to estimate the stellar continuum level at the wavelengths of these lines. Instead, the apparent continuum was fixed locally using polynomials, as illustrated in Fig. 4b. Two components are needed to fit the Fe II lines in the HD 250550 spectrum. Lines from the ground and excited levels up to $3117.46 \mathrm{~cm}^{-1}$ are observed for one component, while only transitions from levels up to $667.68 \mathrm{~cm}^{-1}$ are detected for the second one. In a few cases, as for levels at $2430.10 \mathrm{~cm}^{-1}$ and $2837.95 \mathrm{~cm}^{-1}$, the column densities 

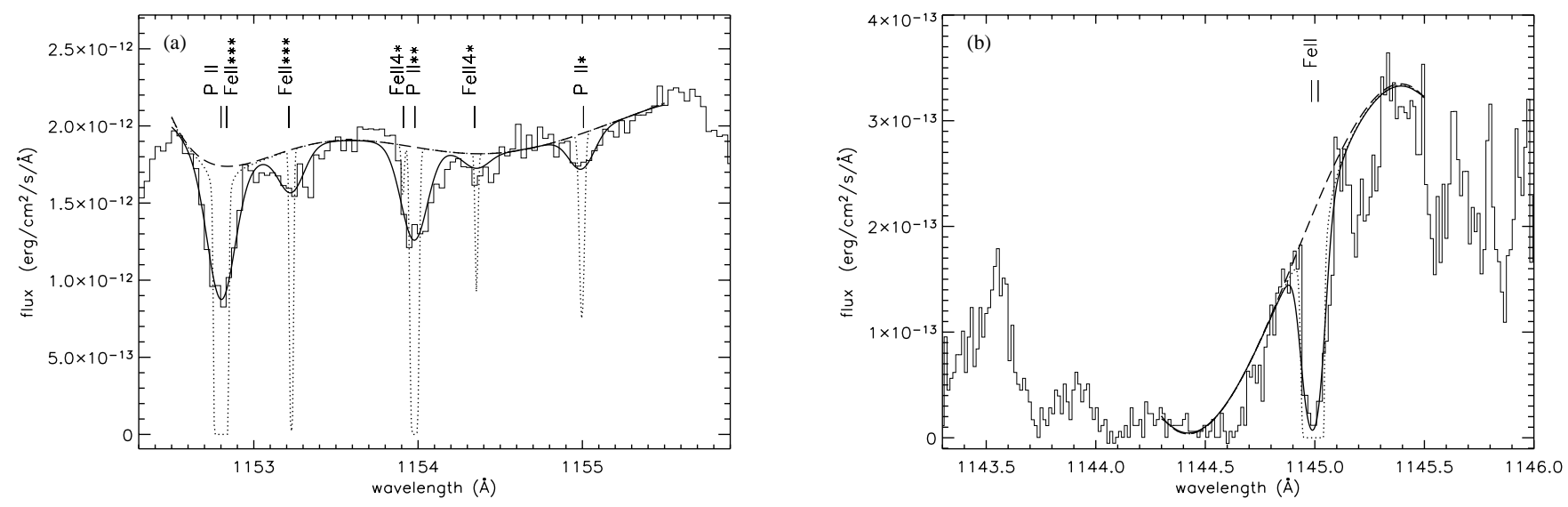

Fig. 4. Examples of fits of lines from atomic species. The observed spectra (thin line) are not corrected for reddening. The OWENS profile fitting procedure fits the stellar continuum (dashed line) by a polynomial, the intrinsic line profile (dotted line), and the resulting profile (thick line). a) LiF1B spectrum of HD 259431 around $1154 \AA$. Note that several species and different excitation states can be fitted in the same spectral window. b) LiF2A segment of HD 250550 around $1145 \AA$. The Fe II lines are blended with the red wing of a prominent wind feature that extends blueward, up to $-350 \mathrm{~km} \mathrm{~s}^{-1}$ (see Fig. 3).

could not be derived properly, as most of the corresponding lines are saturated.

- Fe III - The resonance line at $1122.53 \AA$ falls at the bottom of a strong absorption feature and is not observed. On the other hand, lines from excited levels are clearly detected. For levels at $436.20 \mathrm{~cm}^{-1}$ and $1027.30 \mathrm{~cm}^{-1}$, the column densities of Fe III could not be reliably estimated, the majority of the lines from these levels being saturated. Same comments as for HD 259431 concerning the collisional origin of this ion.

\subsection{Atomic and molecular hydrogen}

The FUSE spectral range contains the Lyman $(\mathrm{B} \rightarrow \mathrm{X})$ and Werner $(\mathrm{C} \rightarrow \mathrm{X})$ systems of molecular hydrogen, which all lie in the spectral region between 844 and $1126 \AA$. In this wavelength band, the observed lines correspond to electronic transitions. As expected, the far-UV spectra of our stars, which are significantly reddened, exhibit a wealth of $\mathrm{H}_{2}$ absorption lines, although the contributions from molecular hydrogen differ widely from one star to the other.

Column densities for individual levels were measured with the program OWENS. For each spectral window of interest, we fit all available lines arising from all or any $J$-levels simultaneously. This serves to decrease the errors which arise in the continuum level because of overlapping wings of adjacent lines (see Fig. 5). 2- $\sigma$ error bars on column densities were estimated through a $\chi^{2}$ procedure. Results from this fitting process are tabulated in Table 3 .

\subsubsection{HD 259431}

We have identified $\mathrm{H}_{2}$ lines corresponding to $J=0$ to $J=9$ in the ground vibrational state, with a line with $J=10$ marginally detected. Moreover, transitions arising from various rotational levels (as high as $J=5$ ) in the first excited vibrational state of the ground electronic state are also detected.
As far as atomic hydrogen is concerned, we took advantage of IUE spectra to model the Ly $\alpha$ line. Despite the presence of a strong contamination by geocoronal Ly $\alpha$ emission, we see some weak emission at the bottom of the observed Ly $\alpha$ line. This emission is redshifted with respect to the rest wavelength $\left(\Delta \lambda=0.52 \AA\right.$, i.e. $\left.\Delta V \approx 130 \mathrm{~km} \mathrm{~s}^{-1}\right)$ and most likely corresponds to the red component of a double-peaked profile produced in the rotating chromosphere of the star. The expected blue component in Ly $\alpha$ is not seen because it is blended with geocoronal emission. The emission is weak enough that we can safely neglect it when measuring the column density of interstellar neutral hydrogen; using our photospheric model, we find $N(\mathrm{HI})=1.6 \times 10^{21} \mathrm{~cm}^{-2}$. This result was checked and confirmed by using the IUE spectrum of a main-sequence analog, HD 25340, as a template instead of our model. As expected, the derived $N(\mathrm{HI})$ is insensitive to the adopted $b$-value.

\subsubsection{HD 250550}

$\mathrm{H}_{2}$ lines are detected up to $v=0, J=4$. Higher rotational $J$-levels and vibrational levels are beyond the detection limit, because of the steep decrease in flux and lower signal-to-noise ratio of the data caused by the later spectral type of this star. The low signal-to-noise ratio is responsible for the rather high uncertainty in the determination of the column density of these levels.

Quite surprisingly for a star showing C III or even $\mathrm{O}$ VI lines, Ly $\alpha$ emission is not detected in high resolution IUE spectra (Blondel et al. 1993). This might be an observational bias, since, for stars earlier than A1 and later than B3, the stellar continuum flux is bright enough around $1215 \AA$ to hinder the detection of Ly $\alpha$ emission. Yet, as pointed out by Blondel et al. (1993), the fact that no Ly $\alpha$ emission is detected means that chromospheric Ly $\alpha$ photons are fully absorbed in the extended CS envelope formed by the stellar wind. The modelling of HD 250550 (see Sect. 3.3) helps establish the total interstellar absorption due to hydrogen, $\mathrm{N}(\mathrm{H} \mathrm{I})=9.0 \times 10^{20} \mathrm{~cm}^{-2}$. Here again, the column density is insensitive to the adopted $b$-value 
Table 2. Summary of the column densities of various the atomic species present in the spectra of HD 259431 and HD 250550 and analysed with OWENS.

\begin{tabular}{|c|c|c|c|}
\hline & & HD 259431 & HD 250550 \\
\hline Element & $\begin{array}{c}\text { Energy levels } \\
\left(\mathrm{cm}^{-1}\right)\end{array}$ & $\begin{array}{c}N \\
\left(\mathrm{~cm}^{-2}\right)\end{array}$ & $\begin{array}{c}N \\
\left(\mathrm{~cm}^{-2}\right)\end{array}$ \\
\hline N I & 0.00 & $-{ }^{b}$ & $-^{b}$ \\
\hline N I & 19224.46 & $-^{a}$ & $4.37_{-2.06}^{+2.29} \times 10^{15}$ \\
\hline $\mathrm{N} \mathrm{I}$ & 28838.92 & $-^{a}$ & $2.19_{-0.68}^{+0.00} \times 10^{15}$ \\
\hline O I & 0.00 & $-{ }^{b}$ & $\begin{array}{r}-0.08 \\
-a\end{array}$ \\
\hline O I & 158.26 & $-^{b}$ & $-^{a}$ \\
\hline O I & 226.98 & $-^{b}$ & $-^{a}$ \\
\hline O I & 15867.86 & $-{ }^{b}$ & $-^{a}$ \\
\hline P II & 0.00 & $1.78_{-124}^{+1.20} \times 10^{16}$ & $7.59_{-277}^{+1.66} \times 10^{13}$ \\
\hline P II & 164.9 & $2.69_{-1.08}^{+1.124} \times 10^{13}$ & $2.88_{-0.55}^{+1.49} \times 10^{13}$ \\
\hline P II & 469.12 & $3.63_{-1.99}^{+1.90} \times 10^{13}$ & $1.00_{-0.76}^{+0.85} \times 10^{14}$ \\
\hline $\mathrm{Cl} \mathrm{I}$ & 0.00 & -1.99 & $1.58_{-0.73}^{+0.55} \times 10^{14}$ \\
\hline $\mathrm{Cl} \mathrm{I}$ & 882.35 & $-^{a}$ & $1.26_{-0.33}^{+0.23} \times 10^{14}$ \\
\hline $\mathrm{Cl} \mathrm{II}$ & 0.00 & $-^{a}$ & $1.00_{-0.26}^{+0.95} \times 10^{15}$ \\
\hline $\mathrm{Cl}$ II & 696.00 & $-^{a}$ & $1.51_{-0.73}^{+0.05} \times 10^{15}$ \\
\hline $\mathrm{Cl}$ II & 996.47 & $-^{a}$ & $1.58_{-0.27}^{+1.04} \times 10^{14}$ \\
\hline Ar I & 0.00 & $-{ }^{b}$ & $3.98_{-3.76}^{+0.67} \times 10^{15}$ \\
\hline Mn II & 0.00 & $4.79_{-1.34}^{+2.21} \times 10^{14}$ & $1.58_{-0.45}^{+0.98} \times 10^{15}$ \\
\hline Fe II & 0.00 & $1.99_{-0.96}^{+1.01} \times 10^{15}$ & $\begin{array}{l}2.88_{-2.00}^{+1.55} \times 10^{17}(1) \\
3.02_{-1.32}^{+0.67} \times 10^{17}(2)\end{array}$ \\
\hline $\mathrm{Fe}$ II & 384.79 & $1.91_{-1.05}^{+1.00} \times 10^{14}$ & $\begin{array}{l}1.58_{-0.89}^{+0.30} \times 10^{15} \\
1.86_{-0.76}^{+0.56} \times 10^{14}\end{array}$ \\
\hline $\mathrm{Fe}$ II & 667.68 & $1.70_{-0.51}^{+0.59} \times 10^{14}$ & $\begin{array}{l}1.26_{-0.22}^{+0.61} \times 10^{16}(1) \\
3.89_{-1.27}^{+0.46} \times 10^{15}\end{array}$ \\
\hline $\mathrm{Fe}$ II & 862.61 & $1.26_{-0.51}^{+0.28} \times 10^{14}$ & $1.20_{-0.66}^{+0.30} \times 10^{14}$ \\
\hline $\mathrm{Fe}$ II & 977.05 & $3.98_{-1.85}^{+3.96} \times 10^{13}$ & $1.00_{-0.18}^{+0.21} \times 10^{15}$ \\
\hline $\mathrm{Fe}$ II & 1872.57 & $-{ }^{a}$ & $1.91_{-0.61}^{+0.18} \times 10^{15}$ \\
\hline $\mathrm{Fe}$ II & 2430.10 & $-^{a}$ & $-{ }^{b}(1)$ \\
\hline Fe II & 2837.95 & $-^{a}$ & $-^{b(1)}$ \\
\hline $\mathrm{Fe}$ II & 3117.46 & $-^{a}$ & $1.66_{-0.59}^{+0.26} \times 10^{15}$ \\
\hline $\mathrm{Fe}$ III & 0.00 & $-{ }^{b}$ & -0.59 \\
\hline $\mathrm{Fe}$ III & 436.20 & $-^{b}$ & $-^{b}$ \\
\hline $\mathrm{Fe}$ III & 738.88 & $6.76_{-4.66}^{+2.28} \times 10^{14}$ & $3.55_{-2.05}^{+1.03} \times 10^{15}$ \\
\hline $\mathrm{Fe}$ III & 932.40 & $2.51_{-0.99}^{-1.32} \times 10^{14}$ & $2.00_{-0.64}^{+0.05} \times 10^{14}$ \\
\hline $\mathrm{Fe}$ III & 1027.30 & $2.63_{-1.36}^{+3.85} \times 10^{13}$ & $-b$ \\
\hline
\end{tabular}

and is unchanged if we use a main-sequence star template observation instead of a theoretical model.

\section{Discussion}

Results from the analysis presented in the previous sections provide us with the fundamental quantities required to determine the total column density of hydrogen atoms $N_{\mathrm{H}}=$ $N(\mathrm{H} \mathrm{I})+2 N\left(\mathrm{H}_{2}\right)$. We obtain $N_{\mathrm{H}}(\mathrm{HD} 259431)=2.5 \times 10^{21} \mathrm{~cm}^{-2}$ and $N_{\mathrm{H}}(\mathrm{HD} 250550)=9.4 \times 10^{20} \mathrm{~cm}^{-2}$. These column densities
Table 3. $\mathrm{H}_{2}$ column densities with $2-\sigma$ error bars.

\begin{tabular}{lccc}
\hline \hline & & HD 259431 & HD 250550 \\
\hline $\mathrm{H}_{2}$ & $v$ & $\begin{array}{c}N \\
\left(\mathrm{~cm}^{-2}\right)\end{array}$ & $\begin{array}{c}N \\
\left(\mathrm{~cm}^{-2}\right)\end{array}$ \\
\hline$J=0$ & 0 & $2.51_{-0.77}^{+0.89} \times 10^{20}$ & $4.71_{-2.77}^{+6.28} \times 10^{18}$ \\
$J=1$ & 0 & $1.78_{-0.66}^{+0.91} \times 10^{20}$ & $1.29_{-0.79}^{+0.24} \times 10^{19}$ \\
$J=2$ & 0 & $5.01_{-2.01}^{+1.91} \times 10^{18}$ & $4.90_{-2.89}^{+1.83} \times 10^{17}$ \\
$J=3$ & 0 & $7.02_{-3.04}^{+2.98} \times 10^{16}$ & $3.89_{-1.98}^{+4.92} \times 10^{16}$ \\
$J=4$ & 0 & $8.96_{-2.19}^{+9.19} \times 10^{14}$ & $1.82_{-1.20}^{+2.17} \times 10^{15}$ \\
$J=5$ & 0 & $5.70_{-1.91}^{+1.82} \times 10^{14}$ & \\
$J=6$ & 0 & $5.51_{-2.35}^{+1.17} \times 10^{14}$ & \\
$J=7$ & 0 & $1.42_{-0.79}^{+0.57} \times 10^{14}$ & \\
$J=8$ & 0 & $3.41_{-3.28}^{+2.32} \times 10^{13}$ & \\
$J=9$ & 0 & $6.25_{-4.36}^{+6.01} \times 10^{13}$ & \\
$J=0$ & 1 & $2.82_{-0.90}^{+0.91} \times 10^{13}$ & \\
$J=1$ & 1 & $1.17_{-0.48}^{+0.10} \times 10^{14}$ & \\
$J=2$ & 1 & $1.05_{-0.37}^{+0.22} \times 10^{14}$ & \\
$J=3$ & 1 & $1.70_{-0.52}^{+0.45} \times 10^{14}$ & \\
$J=4$ & 1 & $1.23_{-0.47}^{+0.26} \times 10^{14}$ & \\
$J=5$ & 1 & $9.12_{-2.91}^{+0.27} \times 10^{13}$ & \\
\hline Total $\mathrm{H}_{2}$ & $(0)$ & $4.34_{-1.55}^{+1.23} \times 10^{20}$ & $1.81_{-1.09}^{+0.89} \times 10^{19}$ \\
Total $\mathrm{H}_{2}$ & $(1)$ & $6.34_{-2.22}^{+1.15} \times 10^{14}$ & \\
\hline
\end{tabular}

are somewhat higher than the usual ones for diffuse molecular clouds but are compatible with typical values for circumstellar envelopes.

The molecular hydrogen abundance is sensitive to the formation/destruction equilibrium of $\mathrm{H}_{2}$ in diffuse gas, where FUV radiation competes with the formation of dust grains to determine the relative abundances of $\mathrm{H}_{2}$. In terms of the total column densities of $\mathrm{HI}$ and $\mathrm{H}_{2}$, the fraction of hydrogen atoms in molecular form is given by:

$f\left(\mathrm{H}_{2}\right)=2 N\left(\mathrm{H}_{2}\right) /\left(2 N\left(\mathrm{H}_{2}\right)+N(\mathrm{H} \mathrm{I})\right)$.

This quantity directly expresses the number of hydrogen nucleii bound into $\mathrm{H}_{2}$. Therefore the observed molecular fraction is a measure of interaction between formation and destruction, and it can be modified both by reduced formation rate on dust grains and by enhanced photo-dissociating radiation.

For HD 259431 and HD 250550 we find that $f_{\mathrm{H} 2}=0.35$ and $f_{\mathrm{H} 2}=0.04$, respectively. In the first case, $f_{\mathrm{H} 2}$ is slightly lower than values found for stars with similar $E(B-V)$ that have been observed with COPERNICUS or FUSE (e.g., Savage et al. 1977; Gry et al. 2002), while it is suprisingly low for a late Herbig Be star like HD 250550. Note that for the A0 V star AB Aur, Roberge et al. (2001) measured a molecular hydrogen fraction $f_{\mathrm{H} 2}=0.31$, which is very close to our estimate for HD 259431. The much lower molecular fraction in HD 250550 may indicate a less efficient formation of $\mathrm{H}_{2}$ molecules on dust grains in the CS environment of this star, as photo-dissociation is less efficient in HD 250550 than in the hotter HD 259431. A quantitative modelling, using the methods described by Gry et al. (2002) or Tumlinson et al. (2002) would allow further investigation of this issue, but is far beyond the scope of the present paper. 


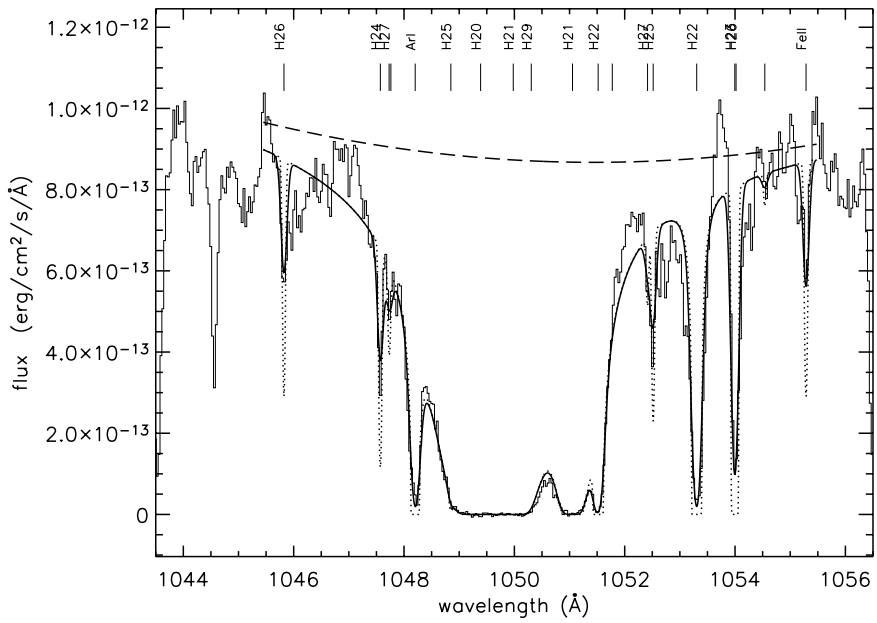

Fig. 5. Example of fit of $\mathrm{H}_{2}$ lines in the observed spectrum of HD 259431 (solid, thin line). The OWENS profile fitting procedure fits the stellar continuum (dashed line), the intrinsic line profile (dotted line), and the resulting profile (thick line). Up to nine lines are fitted together on this plot. The $\mathrm{H}_{2}$ levels are marked $\mathrm{H} 20$ for $(v=0, J=0)$, $\mathrm{H} 21$ for $(v=0, J=1)$ etc. Ar I and Fe II lines are also present in this range and are labeled at the top of the plot.

\section{1. $H D 259431$}

Figure 6 presents the excitation diagram corresponding to the $\mathrm{H}_{2}$ column densities derived from our FUSE spectrum of HD 259431. This plot shows that three temperatures are needed to fit the measurements. The $v=0$ levels up to $J=3$ are consistent with a kinetic temperature of $T_{03}=92 \pm 5 \mathrm{~K}$, which is higher than the typical temperature of the diffuse IS medium (Gry et al. 2002). Higher $v=0$ levels, with $4 \leq J \leq 9$, and the vibrationally excited $v=1$ levels are compatible with thermal equilibrium at much higher temperatures of $T_{49}=1312 \pm$ $250 \mathrm{~K}$ and $T_{(v=1)}=1329 \pm 280 \mathrm{~K}$, respectively. Note that the very presence of absorption lines of vibrationally excited levels in the spectrum of a mid-B star is generally regarded as an indication that warm $\mathrm{H}_{2}$ is present on the line of sight.

These results favour an interpretation in terms of multitemperature components for the gas, much like what was found for the later A0 V star AB Aur on the basis of near-IR observations of the $\mathrm{CO}$ molecule (Brittain et al. 2003). According to these authors, the $\mathrm{CO}$ emission is best interpreted as coming from two places in a flared CS disk around the star: (i) a hot gas component $(T \approx 1500 \mathrm{~K})$ at the inner rim of the disk, i.e., beyond 0.5 AU from the star; (ii) a cold gas component $(T \approx 100 \mathrm{~K})$ located at distances $\geq 8 \mathrm{AU}$ and farther out from the star. We refer to this paper for details about the data and their interpretation in the framework of the flared-disk model of Dullemond et al. (2001). It is also worth mentioning that the mid-IR observations of HD 259431 by Polomski et al. (2002) are consistent with a model of a moderately flared CS disk. These authors also concluded that the large-scale extended emission they observe likely originates from transiently heated dust grains. The mean temperature they measured for the surrounding dust envelope (over $1700 \mathrm{AU}$ ) is $270 \mathrm{~K}$.

Scaling the Dullemond et al. (2001) results to HD 259431 luminosity, we find that the two hot components of $\mathrm{H}_{2}$ should

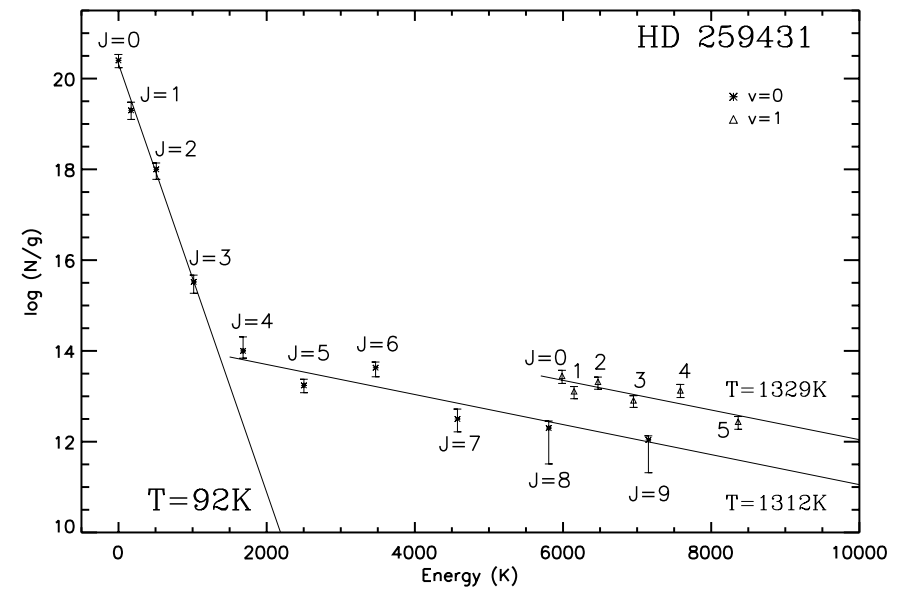

Fig. 6. Excitation diagram for $\mathrm{H}_{2}$ in HD 259431. The slope for the column densities of the low $J$-levels (from $J=0$ to $J=3$ ) is much steeper than for the higher $(v=0, J=4-9)$ levels and vibrationnally excited levels. This reveals both cold and warm components with three different kinetic temperatures (see text).

be located within 3.8 AU from the star while the cold component should be at and beyond $43 \mathrm{AU}$. The moderate velocity shifts (from $+13 \mathrm{~km} \mathrm{~s}^{-1}$ to $+18 \mathrm{~km} \mathrm{~s}^{-1}$, see Table 4) we observe in the $\mathrm{H}_{2}$ lines are compatible with this interpretation.

Concerning the atomic species, the scatter of the radial velocities (see Table 4) is such that any conclusion about the CS/IS nature of the gas is bound to be tentative. Many of these species, like Fe II, Fe III and P II, show several absorption lines corresponding to electronic transitions from fine structure levels. This favors a CS (warm/hot medium) rather than an IS (cold medium) origin for this component, despite the moderate redshift by about $15 \mathrm{~km} \mathrm{~s}^{-1}$ with respect to the star. Furthermore, we have checked that these species cannot originate from a HII region in the vicinity of HD 259431 since data from the ESO Online Digitized Sky Survey show no such $\mathrm{H}$ II region towards the star. This further strengthens a CS origin for the atomic gas component.

\section{2. $H D 250550$}

The excitation diagram of $\mathrm{H}_{2}$ plotted in Fig. 7 shows that the $J$-levels are thermally populated up to $J=3$. The column densities of the thermalized levels are consistent with a single temperature of $T_{03}=129 \pm 15 \mathrm{~K}$. This value is slightly higher than what we found for HD 259431 for the same levels, and up to twice what was found for AB Aur (Roberge et al. 2001). The remaining $J=4$ level departs from kinetic populating at this temperature. This level could be thermalized at a higher temperature in a multi-temperature scenario or populated through UV-pumping. Spectra with higher $\mathrm{S} / \mathrm{N}$ ratio would be required to search for higher $J$-levels in order to constrain the excitation processes.

Assuming that $\mathrm{H}_{2}$ is essentially thermalized at $T_{03}$, we can use the critical density by Le Bourlot et al. (1999) to derive a volume density for this gas: $n_{\mathrm{H}_{2}}(\mathrm{HD} 250550) \gtrsim 1.9 \times 10^{5} \mathrm{~cm}^{-3}$. The ratio of the measured $\mathrm{H}_{2}$ column density to this volume density yields an estimate of the typical radial extension of the 
Table 4. Estimations of total column densities, radial velocities and line widths of each element identified in HD 259431 and HD 250550 FUSE spectra. Radial velocities are given in the stellar rest frame.

\begin{tabular}{lcccccc}
\hline \hline & \multicolumn{3}{c}{ HD 259431 } & \multicolumn{3}{c}{ HD 250550 } \\
\hline Element & $\begin{array}{c}N \\
\left(\mathrm{~cm}^{-2}\right)\end{array}$ & $\begin{array}{c}V_{\text {rad }} \\
\left(\mathrm{km} \mathrm{s}^{-1}\right)\end{array}$ & $\begin{array}{c}b \\
\left(\mathrm{~km} \mathrm{~s}^{-1}\right)\end{array}$ & $\begin{array}{c}N \\
\left(\mathrm{~cm}^{-2}\right)\end{array}$ & $\begin{array}{c}V_{\text {rad }} \\
\left(\mathrm{km} \mathrm{s}^{-1}\right)\end{array}$ & $\begin{array}{c}b \\
\left(\mathrm{~km} \mathrm{~s}^{-1}\right)\end{array}$ \\
\hline $\mathrm{H}_{2}(v=0)$ & $4.34_{-1.55}^{+1.23} \times 10^{20}$ & $13.0_{-1.8}^{+2.2}$ & $4.5_{-0.5}^{+0.3}$ & $1.81_{-1.09}^{+0.89} \times 10^{19}$ & $-1.0_{-2.0}^{+1.7}$ & $5.6_{-0.8}^{+0.7}$ \\
$\mathrm{H}_{2}(v=1)$ & $6.34_{-2.22}^{+1.15} \times 10^{14}$ & $16.0 \pm 0.1$ & $4.5_{-0.5}^{+0.3}$ & & & \\
Fe II & $2.52_{-1.19}^{+1.23} \times 10^{15}$ & $14.4_{-1.8}^{+1.1}$ & $6.6_{-1.1}^{+1.0}$ & $3.07_{-2.05}^{+1.63} \times 10^{17}(1)$ & $-0.6_{-1.9}^{+0.7}(1)$ & $3.6_{-0.6}^{+2.6(1)}$ \\
& & & & $3.06_{-1.33}^{+0.68} \times 10^{17}(2)$ & $-5.2_{-3.5}^{+0.4}(2)$ & $6.3_{-0.5}^{+1.8}(2)$ \\
Fe III & $9.53_{-5.78}^{+3.97} \times 10^{14}$ & $18.0_{-4.9}^{+6.5}$ & $4.3_{-0.5}^{+0.4}$ & $3.75_{-2.11}^{+1.08} \times 10^{15}$ & $4.4_{-2.4}^{+2.7}$ & $3.8_{-0.8}^{+0.9}$ \\
N I & & $15.0 \pm 2$ & $5.5_{-0.8}^{+0.4}$ & $6.56_{-2.74}^{+2.60} \times 10^{15}$ & $3.2_{-1.1}^{+2.9}$ & $6.0_{-0.3}^{+1.4}$ \\
P II & $1.79_{-1.25}^{+1.20} \times 10^{16}$ & $13.0_{-4.2}^{+4.3}$ & $4.5_{-0.5}^{+0.4}$ & $2.05_{-1.09}^{+1.16} \times 10^{14}$ & $3.6_{-2.3}^{+3.2}$ & $6.3_{-1.6}^{+0.4}$ \\
Mn II & $4.79_{-1.34}^{+2.21} \times 10^{14}$ & $17.0_{-3.7}^{+2.8}$ & & $1.58_{-0.45}^{+0.98} \times 10^{15}$ & $1.2_{-2.7}^{+1.5}$ & \\
Ar I & & & $3.5_{-0.1}^{+0.1}(1)$ & $3.98_{-3.76}^{+0.69} \times 10^{15}$ & $-7.6_{-1.9}^{+0.8}$ & $4.5_{-1.4}^{+1.6}$ \\
& & & $6.5_{-0.2}^{+0.3}(2)$ & & & \\
O I & & $5.1_{-0.7}^{+0.8}(1)$ & & & & \\
& & $4.5_{-0.3}^{+0.2}(2)$ & & $2.84_{-1.06}^{+0.78} \times 10^{14}$ & $3.0_{-3.3}^{+2.8}$ & $2.2_{-0.7}^{+0.4}$ \\
Cl I & & & & $2.67_{-1.02}^{2+10} \times 10^{15}$ & $1.0_{-0.9}^{+1.1}$ & $4.8_{-0.4}^{+0.6}$ \\
Cl II & & &
\end{tabular}

(1) First component from the fitting process (see text for details).

(2) Second component from the fitting process.

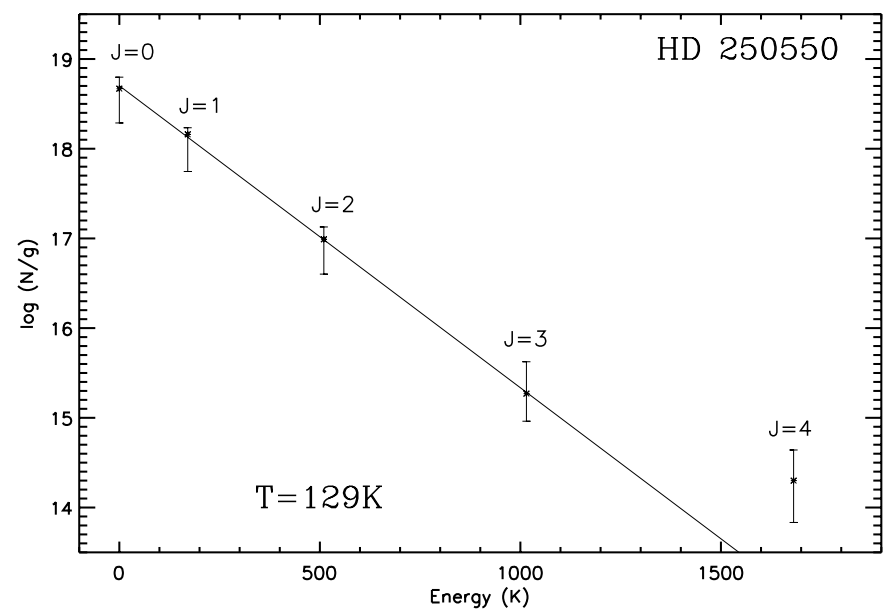

Fig. 7. Excitation diagram for $\mathrm{H}_{2}$ in HD 250550. $J=4$ level depart from a kinetic population at $T_{03} \sim 130 \mathrm{~K}$.

region containing $\mathrm{H}_{2}$. We find that this absorbing region extends roughly over $7 \mathrm{AU}$. The fact that the radial velocity of $\mathrm{H}_{2}$ is very close to the stellar velocity $\left(\approx-1 \mathrm{~km} \mathrm{~s}^{-1}\right)$ is a strong clue that the molecular hydrogen we observe is bound to the star. These properties indicate that we are probing a dense circumstellar environment which is a remnant of the molecular cloud that collapsed to form the star. Similarly, all the atomic species detected in the FUSE spectrum have their radial velocities close to that of HD 250550 (see Table 4), within the uncertainty in the wavelength calibration. Since they also show lines arising from excited levels, which are rarely found in the IS medium (especially for Fe II and Fe III), this further confirms that the gas must be in the close vicinity of the star.
These results concerning the absorption lines arising from the atomic species and $\mathrm{H}_{2}$ are hints that the gaseous material we detect is actually orbiting the central star. Indeed, whether the gas is inside a CS disk or an envelope, the observed species are expected to have their absorption lines observed very close to the radial velocity of the star, which is clearly the case for HD 250550. On the other hand, the issue regarding the spatial distribution of this material is far from being settled. Spectro-polarimetric observations of HD 250550 by Vink et al. (2002) show that a rotating flattened structure exists around the star, much like those observed for AB Aur, for which unambiguous detections have been reported by Mannings \& Sargent (1997); Grady et al. (1999); Millan-Gabet et al. (1999). On the other hand, repeated observations have failed to detect a CS disk around HD 250550 (Böhm \& Catala 1994; Corcoran \& Ray 1997). These results favour a spatial distribution of the CS material in an extended halo surrounding the star, much like those emphasized for Herbig Be stars by Natta et al. (2000) and Leinert et al. (2001).

\section{Summary and discussion}

The FUSE data we have analysed reveal complex environments around the young Herbig Be stars HD 259431 and HD 250550. In the latter case, emission lines from C III and O VI are observed, showing the presence of very high temperature regions $(\approx 300000 \mathrm{~K})$ around the star. Appreciable absorption lines of $\mathrm{H}_{2}$ are seen in each star, in agreement with their $E(B-V)$, revealing large amounts of this gas on the lines of sight. Similarly, numerous absorption lines from atomic species in different ionization and excitation states are present 
in the spectra. In both cases, these molecular and atomic lines are observed at or near the stars' radial velocities, thus suggesting that they probe the close CS environment around each star.

The observation and measure of the gaseous content, both in molecular and atomic phases is important for our understanding of the evolution of the CS environment of Herbig Ae/Be stars. Indeed, the detection of gas in CS envelopes/halos would confirm the structural differences between Herbig Ae and Herbig Be stars emphasized by Natta et al. (2000). In their study, these authors concluded that Herbig Be stars having stronger radiation fields, their CS environment evolve faster, which especially translates into a rapid depletion of the CS disk material. As a consequence of this faster evolution, the average Herbig Be stars are slightly younger than Herbig Ae stars at the time we observe them. Therefore, it is more likely to find larger amounts of remnant circumstellar material surrounding the stars, since less time is available to accrete or disperse it. In this scenario, it is expected that the CS material is mostly concentrated at the outer edges of the close circumstellar environment and distributed in more or less spherically symmetric envelopes.

In this context, we note that HD 250550 and HD 259431 which are $1 \mathrm{Myr}$ old or younger (see Table 1), are indeed much younger than Herbig Ae stars like AB Aur, HD 163296 and HD 100546 (2.5 Myr, 6 Myr, 10 Myr, respectively) which are surrounded by well detected CS disks. In addition, the media we detect with FUSE in our targets are denser than those observed around these Herbig Ae stars (Roberge et al. 2001; Lecavelier des Etangs et al. 2003). This is consistent with the CS environment picture described by Natta et al. (2000). This would indicate that the material we observe is related to CS halos, like those identified by Leinert et al. (2001) on the basis of near-infrared speckle interferometry.

On the other hand, the multi-component media probed by $\mathrm{H}_{2}$ in $\mathrm{HD} 259431$ are easily interpreted in the framework of the flared disk model by Dullemond et al. (2001), recently adopted to interpret mid-IR observations of the $\mathrm{CO}$ molecule in the prototypical HAeBe star AB Aur (Brittain et al. 2003). They also agree qualitatively with those obtained by Lecavelier des Etangs et al. (2003) for the later Herbig Ae stars HD 100546 and HD 163296, although as already said, larger amounts of CS material are found around our targets. In HD 100546 and HD 163296, the molecular lines observed with FUSE are produced in the CS disks within a few AU at most.

The present results, although favouring the flared disk scenario for HD 259431, on the basis of conspicuous similarities with better documented HAeBes, do not rule out the possibility that we actually detect CS envelopes/halos around both stars. Further observations of these stars, as well as other HAeBes covering the whole class, are required to help answer the longstanding questions of the spatial distribution of the CS material of these objects and whether this material survives long enough for massive protoplanetary cores to form and grow into gas giant planets.

Acknowledgements. This research is based on observations made with the NASA-CNES-CSA Far Ultraviolet Spectroscopic Explorer. FUSE is operated for NASA by the Johns Hopkins University under NASA contract NAS5-32985. We thank Sean Andrews for assistance with the data reductions and David Ehrenreich at Johns Hopkins University for reprocessing the pipeline data for us. Dr. Leonardo Testi is also acknowledged for his help in calculating ages and masses of HD 250550 and HD 259431 from stellar evolution models. Dr. Jacques Le Bourlot is acknowledged for providing his calculations of $\mathrm{H}_{2}$ critical densities to us. T.S. acknowledges support by NASA through the FUSE guest observer program to the University of Hawaii.

\section{References}

Abgrall, H., Roueff, E., \& Drira, I. 2000, A\&AS, 141, 297

Abgrall, H., Roueff, E., Launay, F., Roncin, J. Y., \& Subtil, J. L. 1993a, A\&AS, 101, 273

Abgrall, H., Roueff, E., Launay, F., Roncin, J. Y., \& Subtil, J. L. 1993b, A\&AS, 101, 323

Augereau, J. C., Lagrange, A. M., Mouillet, D., \& Ménard, F. 2001, A\&A, 365, 78

Balakrishnan, N., Forrey, R. C., \& Dalgarno, A. 1999, ApJ, 514, 520

Bertout, C., Robichon, N., \& Arenou, F. 1999, A\&A, 352, 574

Bessell, M. S., Castelli, F., \& Plez, B. 1998, A\&A, 333, 231

Blondel, P. F. C., Talavera, A., \& Tjin A Djie, H. R. E. 1993, A\&A, 268, 624

Boggs, D., \& Böhm-Vitense, E. 1989, ApJ, 339, 209

Böhm, T., \& Catala, C. 1994, A\&A, 290, 167

Böhm, T., \& Catala, C. 1995, A\&A, 301, 155

Bouret, J.-C., \& Catala, C. 1998, A\&A, 340, 163

Bouret, J.-C., Catala, C., \& Simon, T. 1997, A\&A, 328, 606

Brittain, S. D., Rettig, T. W., Simon, T., et al. 2003, ApJ, 588, 535

Cardelli, J. A., Clayton, G. C., \& Mathis, J. S. 1989, ApJ, 345, 245

Corcoran, M., \& Ray, T. P. 1997, A\&A, 321, 189

Dullemond, C. P., Dominik, C., \& Natta, A. 2001, ApJ, 560, 957

Finkenzeller, U. 1985, A\&A, 151, 340

Finkenzeller, U., \& Jankovics, I. 1984, A\&AS, 57, 285

Flower, P. J. 1996, ApJ, 469, 355

Garrison, L. M. 1978, ApJ, 224, 535

Grady, C. A., Imhoff, C. L., \& Bjorkman, K. S. 1988, in A Decade of UV Astronomy with the IUE Satellite, 2, 109

Grady, C. A., Polomski, E. F., Henning, T., et al. 2001, AJ, 122, 3396

Grady, C. A., Woodgate, B., Bruhweiler, F. C., et al. 1999, ApJ, 523, L151

Gry, C., Boulanger, F., Nehmé, C., et al. 2002, A\&A, 391, 675

Hamann, F., \& Persson, S. E. 1992, ApJS, 82, 285

Hillenbrand, L. A., Strom, S. E., Vrba, F. J., \& Keene, J. 1992, ApJ, 397,613

Hubeny, I., \& Lanz, T. 1995, ApJ, 439, 875

Kurucz, R. 1994, CD-ROM No. 19, Cambridge, Mass.: Smithsonian Astrophysical Observatory, 19

Le Bourlot, J., Pineau des Forêts, G., \& Flower, D. R. 1999, MNRAS, 305,802

Lecavelier des Etangs, A., Deleuil, M., Vidal-Madjar, A., et al. 2003, A\&A, accepted

Leinert, C., Haas, M., Ábrahám, P., \& Richichi, A. 2001, A\&A, 375, 927

Lemoine, M., Vidal-Madjar, A., Hébrard, G., et al. 2002, ApJS, 140, 67

Mannings, V., \& Sargent, A. I. 1997, ApJ, 490, 792

Mannings, V., \& Sargent, A. I. 2000, ApJ, 529, 391

Millan-Gabet, R., Schloerb, F. P., Traub, W. A., et al. 1999, ApJ, 513, L131

Morton, D. C. 2000, ApJS, 130, 403

Muzerolle, J., Calvet, N., \& Hartmann, L. 2001, ApJ, 550, 944 
Natta, A., Grinin, V., \& Mannings, V. 2000, Protostars and Planets IV, 559

Palla, F., \& Stahler, S. W. 1993, ApJ, 418, 414

Pantin, E., Waelkens, C., \& Lagage, P. O. 2000, A\&A, 361, L9

Polomski, E. F., Telesco, C. M., Piña, R., \& Schulz, B. 2002, AJ, 124, 2207

Roberge, A., Lecavelier des Etangs, A., Grady, C. A., et al. 2001, ApJL, 551, L97

Savage, B. D., Drake, J. F., Budich, W., \& Bohlin, R. C. 1977, ApJ, 216, 291

Schmidt-Kaler, T. 1982, in Landolt-Börnstein, Numerical Data and Functional Relationships in Science and Technology, ed. K. Schaifers, \& H. H. Voigt (Berlin: Springer-Verlag), 2, 451
Sitko, M. L. 1981, ApJ, 247, 1024

Thé, P. S., Perez, M. R., Voshchinnikov, N. V., \& van den Ancker, M. E. 1996, A\&A, 314, 233

Tumlinson, J., Shull, J. M., Rachford, B. L., et al. 2002, ApJ, 566, 857

Valenti, J. A., Fallon, A. A., \& Johns-Krull, C. M. 2003, ApJS, in press Valenti, J. A., Johns-Krull, C. M., \& Linsky, J. L. 2000, ApJS, 129, 399

van den Ancker, M. E., de Winter, D., \& Tjin A Djie, H. R. E. 1998, A\&A, 330, 145

Vink, J. S., Drew, J. E., Harries, T. J., \& Oudmaijer, R. D. 2002, MNRAS, 337, 356 